\title{
Preparation and Characterization of Polymer Nanocomposites
}

\author{
Pratima Parashar Pandey \\ Applied Sciences, CET, IILM, Greater Noida, India \\ Email: pratimaparashara@rediffmail.com
}

How to cite this paper: Pandey, P.P. (2020) Preparation and Characterization of Polymer Nanocomposites. Soft Nanoscience Letters, 10, 1-15.

https://doi.org/10.4236/snl.2020.101001

Received: January 1, 2020

Accepted: January 28, 2020

Published: January 31, 2020

Copyright $\odot 2020$ by author(s) and Scientific Research Publishing Inc. This work is licensed under the Creative Commons Attribution International License (CC BY 4.0).

http://creativecommons.org/licenses/by/4.0/

\begin{abstract}
The polymer nanocomposites have been the exponentially growing field of research for developing the materials in last few decades. The remarkable improvement in the polymer nanocomposite is found when a small amount of nanosized particles are added to a polymer matrix. The addition of inorganic solid nanoparticles (typically in the form of fibres, flakes, spheres or fine particles) into polymer matrix increases their physical, structural and mechanical properties. Since the polymer-nano composites have been the staple of modern polymer industry, their durability under various environmental conditions and degradability after their service life are also essential fields of research. This leads to focus on preparation \& characterization of polymer nanocomposite. This article is intended to review the status of worldwide research in this aspect. The successful application of nano particles depends upon both the correct preparation techniques followed by testing through characterization. Surface modification can improve the inherent characteristics of the nano particles and serve to prepare nano composites inexistent in nature. Therefore, some tools as their various properties like electrical, optical and morphological can be used to optimize the preparation of polymer nano composites. This chapter will make an overview about different routes to prepare polymer-based nano composites by extrusion, synthesis of nano particles by sol-gel reactions, sputtering and mainly by physical evaporation deposition method.
\end{abstract}

\section{Keywords}

Silver Nano-Particle, Polymer Composite, Electrical Properties, Optical Properties, DSC, FTIR, SEM, Morphology

\section{Introduction}

The Nano Science and Nanotechnology is a multidisciplinary branch, and deals 
with materials and structures having dimensions measured up to billionth of a meter (nanometer). In fact, nanotechnology is the applications of Physics, Chemistry, Biology, Engineering and Technology in one fold. Nowadays, we enter a world where Physics and Chemistry together develop novel properties of matter at nano scale. In Chemistry, these sizes are colloids, micelles, polymer molecules, phase-separated regions of block copolymers and similar structures. Polymer nano composites are, actually, hybrid materials (organic \& inorganic) with at least one dimension of the filler phase less than $100 \mathrm{~nm}$ (nanoparticles) [1]. Nanoparticles are solid particles at the intermediate state which is between atoms/molecules and macroscopic objects. Due to special features like small size, large surface and quantum tunnel effect, the nanoparticles exhibit special physical properties. Therefore, these have huge applications in high density magnetic recording, radar signal absorbing, magnetic fluid, radio wave shielding, precision polishing, optical devices, thermal conducting gel for micro chips, electronic packaging, optoelectronics, high-performance battery, solar cell, efficient catalyst, efficient fire retardant, sensitive components, high-toughness ceramics, the body repairing, cancer therapy, and so on [2]. Silver nanoparticles possess a unique property, because their optical, electrical, and magnetic properties are size and shape dependent. Hence, these have applications as antimicrobial, biosensor materials, composite fibers, cryogenic superconducting materials, cosmetic products, and electronic components. The nano composites are composed by high surface area, nano particles, and their interface defines its final properties. The dispersion and surface of particles together result in special properties. The successful applications of nanoparticles depend upon both the synthesis and the surface modification of these nano composite by enhancing their inherent characteristics to prepare new nano composites [3]. In the field of material science, an organic phase (generally, polymers) is combined with inorganic particles (metal nano particles) with considerable interest in last two to three decades. This arises interest for the addition of well dispersed particles (typically in the form of fibres, flakes, spheres or fine particles) in the polymer matrix and allows the development of new materials with tailored-made properties. Nanocomposites are composed by solid structures dispersed at the nano scale in a matrix, in the case of polymers, typically an organic matrix containing inorganic nanoparticles. The nano phases in the nanocomposite structure may be as: zero-dimensional (embedded cluster), 1D (one-dimension; e.g. nanotubes), 2D (nanoscale coatings) and 3D (embedded network) [4]. Nanocomposites materials can be produced both by complex and simple processes. The various chemical approaches, including chemical reduction by a variety of organic and inorganic reducing agents, electrochemical techniques, physiochemical reduction, and radiolysis are used for the synthesis of silver nanoparticles. In the present article, we will discuss the polymer nanocomposites synthesized via various methods like sol-gel, reduction method, melt-mixing and metal evaporation on substrate in or ex situ. The conventional methods involved chemical agents as- 
sociated with environmental toxicity. There is growing need to produce nanocomposite using environmentally friendly methods. Metal evaporation on polymer substrate is an eco-friendly method used by us [5] [6] [7]. In this chapter, all the available methods for preparation of nano-polymer matrix composites along with our own research experience are mainly introduced to the readers. In order to claim the formation of nano-polymer matrix composites, synthesis, modification of nanoparticles, characterization and applications of nano-polymer matrix composites were significantly discussed.

\section{Various Methodologies}

Metal-polymer composites are very important since these are widely used in industry right from food packing to optical data storage. Metal- polymer interface is very sensitive because both are extremely different materials. The possibility of metal polymer interaction is very weak. Polymer metal composite can be prepared by adding metals/their salts into polymer matrix externally or in situ leading to hybrid materials. These various synthesis techniques are critical to obtain nanomaterials with suitable properties. The synthesis of polymer nanocomposites applies bottom-up or top-down [8]. For optimization, the sizes, shape, volume fraction, interface and degree of dispersion or aggregation needs to be checked. A good selection of the basic components as well the way of preparation allows synergetic properties of the prepared nanocomposites. In this chapter, we have discussed various methods of dispersing metal nano particles into polymer matrix.

\subsection{Chemical Reduction Method}

Polyaniline /silver nanoparticles (PANI-Ag) nanocomposite was synthesized by in-situ polymerization of aniline in the presence of silver nitrate as precursor, a chemical reduction method. Here, Aniline is oxidized with silver nitrate to produce PANI nitrate and metallic silver metal. The conducting Polyaniline is an organic conducting polymer due to its high conductivity with good environmental stability and huge variety of applications. Therefore, it is a suitable polymer as a matrix for preparation of conducting polymer nanocomposites with noble metals, such as silver as fillers, which play a major role in strengthening the properties of nanocomposites. This conducting (PANI-Ag), a hybrid material can behave as semiconductor at low temperature and as metal at high temperature. The samples of this polymer composite were characterized by Ultraviolet-visible spectroscopy (UV-VIS), Photoluminescence (PL), X-ray diffraction (XRD), Fourier Transform Infrared spectroscopy (FTIR), Scanning Electron Microscopy (SEM) (Figures 1(a)-(d)) and Thermo gravimetric analysis (TGA) to study silver nanoparticles embedded into PANI. The formation of single crystalline Ag nanoparticles in the polymer matrix is confirmed by sharp peaks in XRD pattern and the average crystallite size was estimated to be $12 \mathrm{~nm}$ using Scherrer formula. The FTIR spectra show all the bands in PANI structure along 


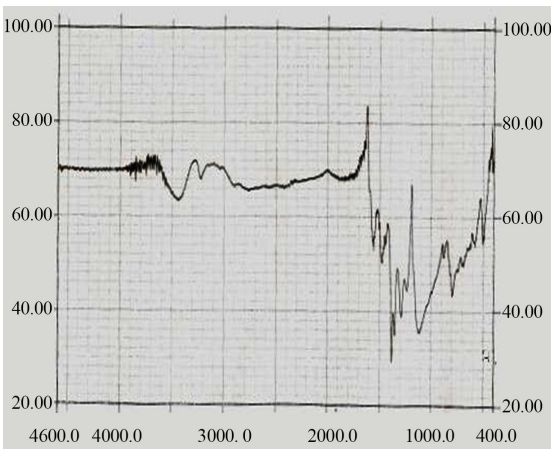

(a)

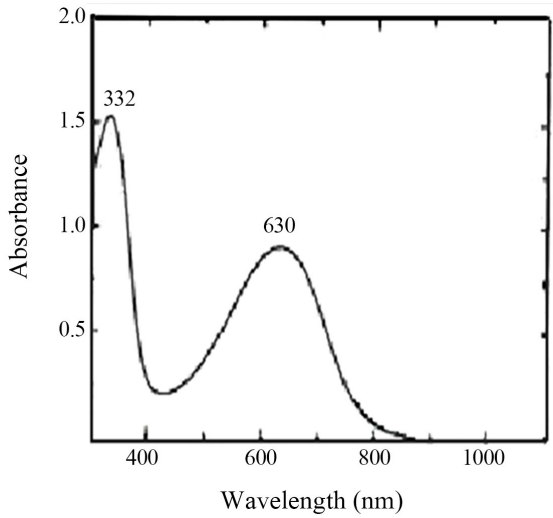

(c)

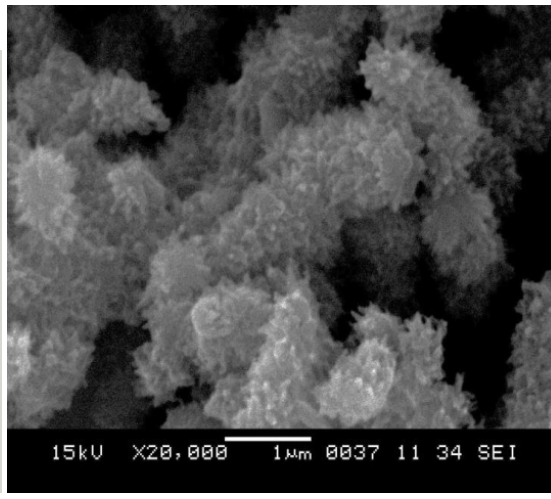

(b)

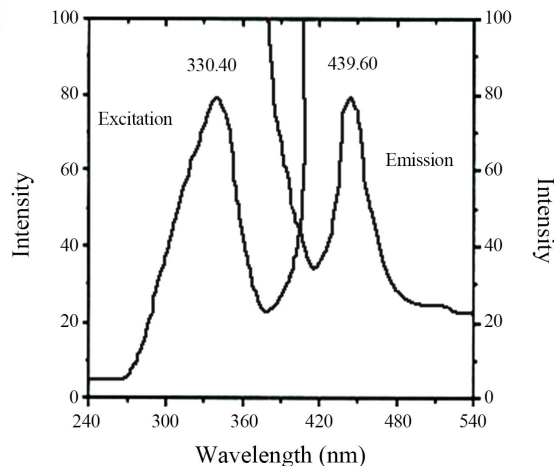

(d)

Figure 1. (a) FT-IR spectra of PANI/Ag nanocomposite; (b). SEM image of PANI/Ag nanocomposite. (c) UV-VIS spectrum of PANI-Ag nanocomposite. (d) PL spectrum of PANI-Ag nanocomposites, Figure 1(a), Figure 1(b), Figure 1(c), Figure 1(d) [9].

with additional bands at 441.8 and $422.5 \mathrm{~cm}^{-1}$ belonging to Ag. UV -visible spectrum of PANI-Ag nanocomposite shows the significant blue-shift which is observed as the quantum confinement of charge carriers in the nanocrystals. The photoluminescence (PL) spectrum of PANI-Ag nonocomposite observed emission wavelength peak at $439.60 \mathrm{~nm}$ shifted towards to the blue region when compared to the bulk PANI-Ag as a result of recombination of electron and hole pairs. The difference between the absorption and emission peak wavelength is $109.20 \mathrm{~nm}$. This indicates the emission associated with the transition of electrons from trap state of conduction band (or valence band). The silver content in residue of thermo gram of PANI-Ag nanocomposites is found. The conductivity of the nanocomposites increases with the increase of temperature until a temperature of around $70^{\circ} \mathrm{C}$ which is a property of semiconductors [9].

\subsection{Electron Beam Evaporation Method}

The other method of producing silver polymer nanocomposites is by electron beam evaporation on Polymethylmethaacrilate and Polyethyleneterephthalate (PMMA-PET). Silver film of thickness of $1,5,10,15$ and $20 \mathrm{~nm}$ by electron beam evaporation at room temperature (residual gas pressure $10-5 \mathrm{~Pa}, T=293$ 
K) with average deposition rate $\sim 0.7 \mathrm{~nm} / \mathrm{s}$ ) are deposited on polymer composite [10]. The characterization of metalized thin films is carried out by X-ray fluorescence spectrometer (XRF), XRD (Figure 2(b)), UV-VIS, Atomic force Microscopy (AFM) and electrical properties. XRF analysis shows silver amount in the polymer structure as discontinuous film till $10 \mathrm{~nm}$ and after that the film becomes continuous (Figure 2(a)). Initial orientations of nuclei are depended on the adsorption energy of adatoms to substrate and bonding energy among them. The Volmer-Weber growth mode is of two types according to wettability of the substrates. Wetting mode nuclei have smaller contact angles and preferential orientations. On the contrary, nuclei of non wetting mode usually orient randomly. Metals of low reactivity as silver do not wet untreated polymer surfaces and exhibit a Volmer-Weber growth mode, i.e., these form clusters on the surface, which finally coalesce at high metal coverage [11]. The results suggest that Ag films grow in wetting mode (or the secondary nucleation take a place during film growth [12]. Deposition of silver on PMMA-PET is in wetting mode. At low thickness the crystallite size of silver is almost equal to film thickness, thereafter, it increases with increasing thickness. The conductivity of Ag films on polymer composite increases with thickness and is about $9.43 \times 10^{3} \Omega^{-1} \cdot \mathrm{cm}^{-1}$ much above typical polymers conductivity values $\left(\sim 5 \times 10^{-15} \Omega^{-1} \cdot \mathrm{cm}^{-1}\right)$. The AFM result suggests decrease in skewness of PET decrease by layer of PMMA followed by silver deposition from 3.74 to $0.85 \mathrm{~nm}$. The decrease in the average roughness and increasing negative skewness values with the increasing film thickness (between the thickness values $5 \mathrm{~nm}-10 \mathrm{~nm}$ ) is related to the lateral island growth on the surface of Ag-PMMA-PET structure, also to the filling by silver atoms the deep valley of polymer matrix (Figure 3 ). The surface plasmon resonance (SPR) absorption peak is near to $425 \mathrm{~nm}$ and shifts to the longer waves (up to $600 \mathrm{~nm}$ ) with thickness, and the band broadening significantly (Figure 4). The spherical particles are observed at lower thickness. Extinction spectra for Ag nanoparticles ( $1 \mathrm{~nm}-5 \mathrm{~nm} \mathrm{Ag}$ thickness) shows NPs embedded in a polymer matrix (Figure 5). According to [9] they have observed "blue shift" with particles roughly spherical (diameters $40 \mathrm{~nm}-90 \mathrm{~nm}$ ) at the low thickness of silver film $(1 \mathrm{~nm})$, "green shift" with typically hexagonal shape (longest dimension $-60 \mathrm{~nm}-105 \mathrm{~nm}$ ), and "red shift" with a triangular cross section (longest dimension $-55 \mathrm{~nm}-120 \mathrm{~nm}$ ) at the higher thickness. According to [13] [14] [15], we observed the non-spherical particles (nanorods, nanowires or nanodisk) with transverse and longitudinal SPR peaks of rod-shape silver nanostructures at $352 \mathrm{~nm}$ and $630 \mathrm{~nm}$. The peak at $414 \mathrm{~nm}$ indicated the formation of silver nanoparticles with a diameter $\sim 30 \mathrm{~nm}$. Table $1 \&$ Table 2 comprises the crystallite size and micro strain of Ag films on PMMA-PET, morphology and grain size of Ag films on PMMA-PET and Ag films on PET, respectively.

\subsection{Co-Condensation (COCON) Technique}

The other way of synthesizing the silver nanoparticles is Co-Condensation (COCON) technique [16]. This method has the advantages of chemical and va- 
cuum routes for synthesis of nanoparticle. This is a combined technique of chemical and vacuum evaporation used to synthesize metal nanoparticles. In general, chemical techniques produce smaller nanoparticles with a narrower particle size distribution, while vacuum techniques produce highly pure nanoparticles. Here, nanoparticles do not have any interaction with oxygen. Otherwise, these get oxidized due to their high surface energy and affinity for oxygen when exposed to atmosphere. To utilize their unique properties, minimization of their interaction with atmosphere is needed. Various techniques like co-condensation, i.e., suspensions of metal nanoparticles in isopropanol minimize agglomeration as well as interaction of the nanoparticles. The COCON technique is the deposition of metal vapors by evaporation, and a suitable solvent cryogenically cooled on walls of an evacuated chamber (Figure 7). The super cooling of the metal vapors form metal nuclei while moving away from the evaporation source. These are deposited on the chamber walls together with the solvent.

Table 1. The crystallite size and micro strain of Ag films on PMMA-PET films on PET.

\begin{tabular}{ccc}
\hline Thickness, $\mathrm{nm}$ & $\mathrm{D}$ (crystallite/domain size), $\mathrm{nm}$ & $\Sigma($ strain $), \%$ \\
\hline 10 & 28.52 & 0.73 \\
15 & 15.32 & 0.85 \\
20 & 32.91 & 1.13 \\
$20 \mathrm{~nm}$ on PET & 11.6 & 0.87 \\
\hline
\end{tabular}

Table 2. Morphology and grain size of Ag films on PMMA-PET and Ag.

\begin{tabular}{ccccccc}
\hline $\begin{array}{c}\text { Para } \\
\text { Meter }\end{array}$ & PMMA-PET & $\begin{array}{c}1 \mathrm{~nm} \\
(\mathrm{Ag})\end{array}$ & $\begin{array}{c}5 \mathrm{~nm} \\
(\mathrm{Ag})\end{array}$ & $\begin{array}{c}10 \mathrm{~nm} \\
(\mathrm{Ag})\end{array}$ & $\begin{array}{c}15 \mathrm{~nm} \\
(\mathrm{Ag})\end{array}$ & $\begin{array}{c}20 \mathrm{~nm} \\
(\mathrm{Ag})\end{array}$ \\
\hline$R a$ & 133.2 & 587.3 & 644.0 & 95.8 & 228.1 & 232.4 \\
$R q$ & 170.1 & 675.5 & 726.6 & 145.1 & 293.5 & 290.9 \\
$R s k$ & -0.74 & -0.75 & -0.64 & -2.42 & -0.05 & -0.40 \\
\hline
\end{tabular}

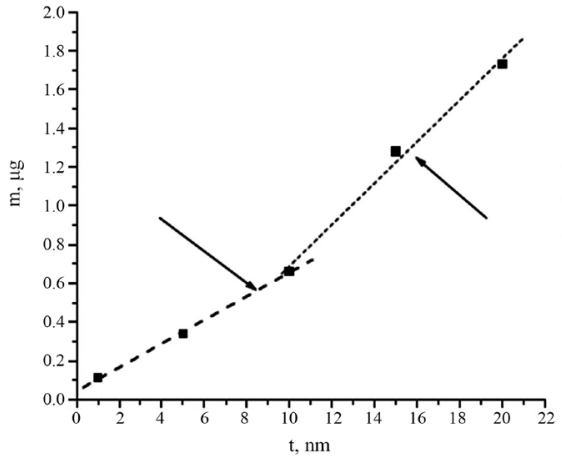

(a)

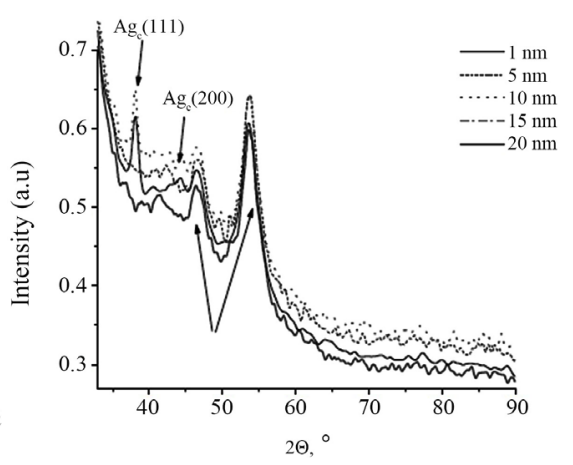

(b)

Figure 2. (a) Dependence of amount of silver in PMMA-PET versus thin layer thickness; (b) XRD pattern of Ag films on PMMA-PET substrate of different thickness. Table \& figures: [10]. 


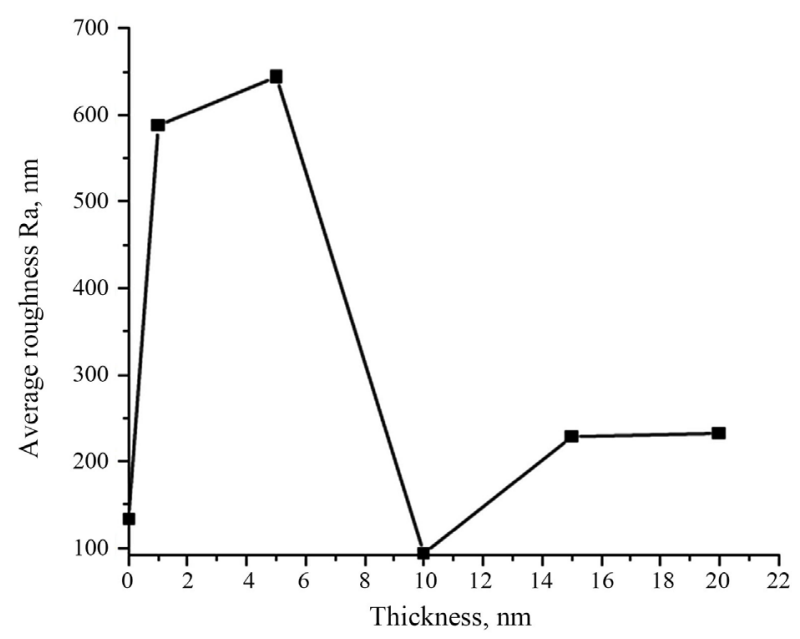

Figure 3. Dependence of the roughness of Ag films on thin film thickness structure.

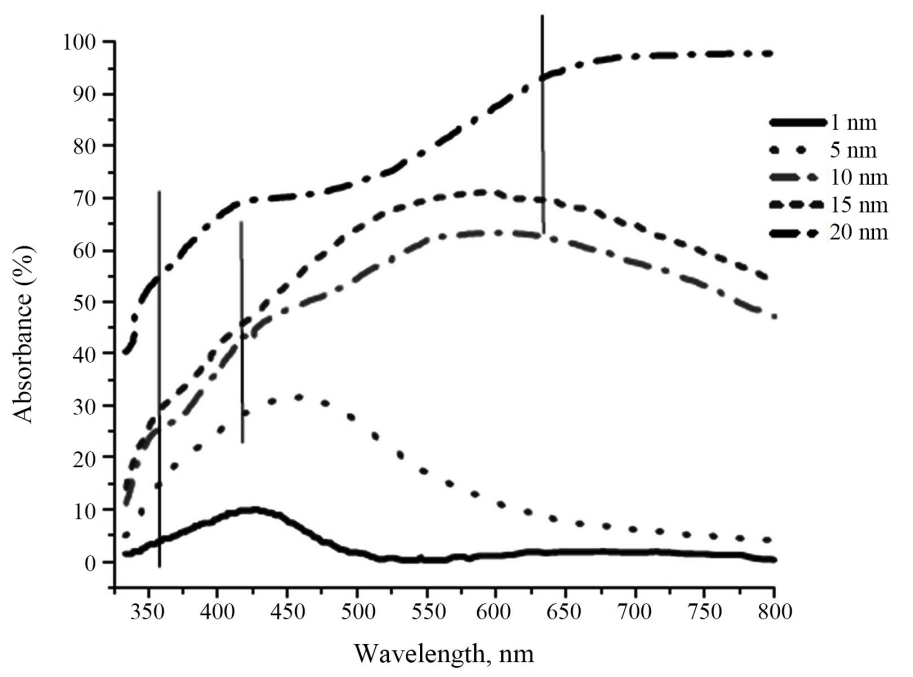

Figure 4. Absorbance spectra of Ag-PMMA-PET.

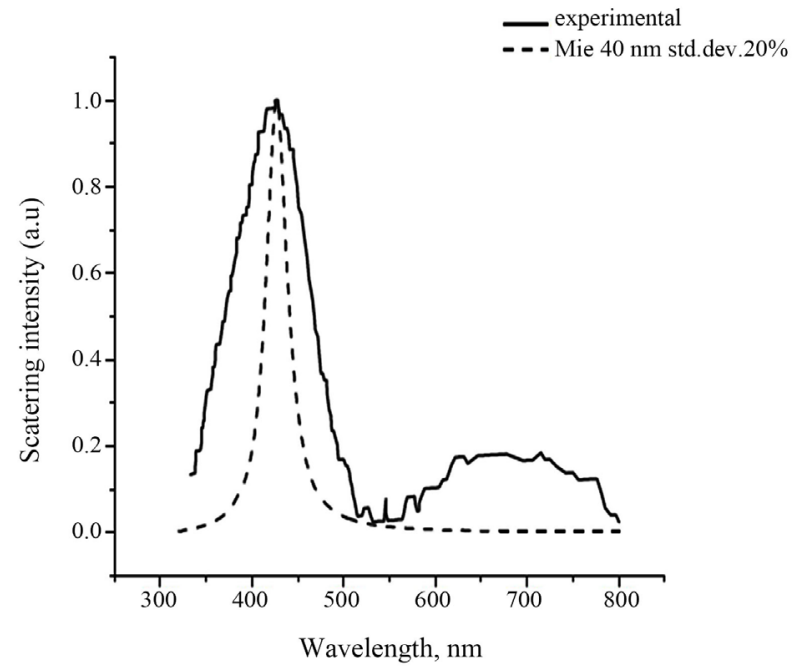

(a) 


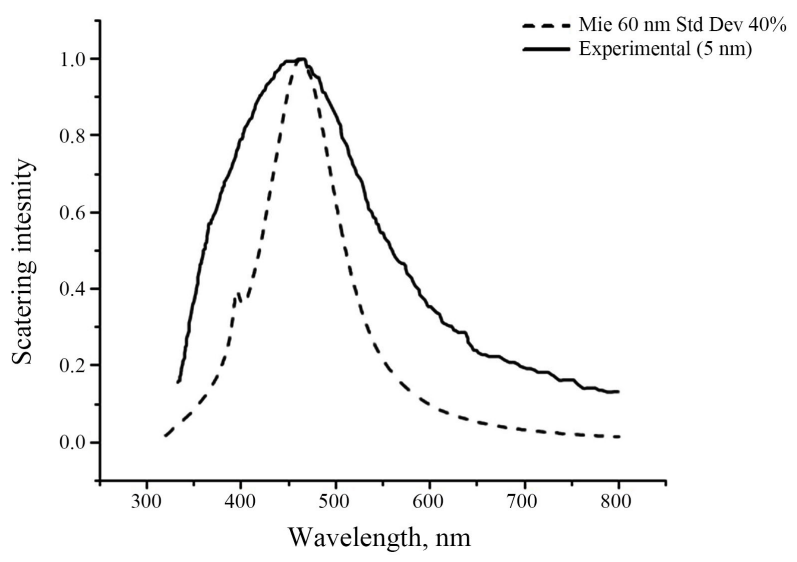

(b)

Figure 5. Spectra of Ag spherical particles in PMMA matrix at different thickness of Ag film: (a) $1 \mathrm{~nm}$ and (b) $5 \mathrm{~nm}$ Figures 3-5: [10].

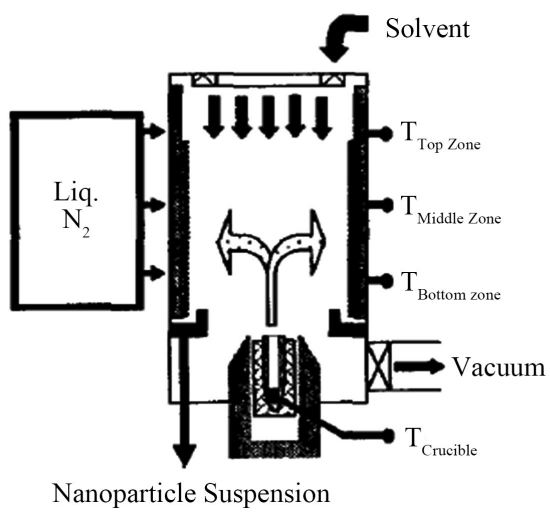

Figure 6. The schematic diagram of COCON process [16].

No growth occurs on cold chamber wall, however, on warming the reactor the nuclei grow to form metal nanoparticles. The solvent molecules surround the growing metal nuclei and prevent agglomeration. This solvation effect is critically important as it not only disperses the nanoparticles within the liquid medium; it also prevents oxidation of the nanoparticles which is a common problem in the synthesis of metal nanoparticles. Figure 6 shows the schematic diagram of COCON process [16].

\subsection{Spin Coating and Solution Cast Method}

In other methods, polymer-metal nanocomposites were made by dispersing the metal nanoparticles in PMMA matrix by spin coating and solution casting [17]. PMMA was chosen since it is a commonly used transparent polymer and is soluble in 1:1 mixture of 2-propanol and MEK (methyl ethyl ketone). In spin coated films nanoparticles are not agglomerated and well dispersed over a wide area. However, morphology of the spin coated films was different from the solution cast films (Figure 7). The morphology is strongly dependent on PMMA-Ag ratio. Films deposited at higher polymer concentrations contain individual 


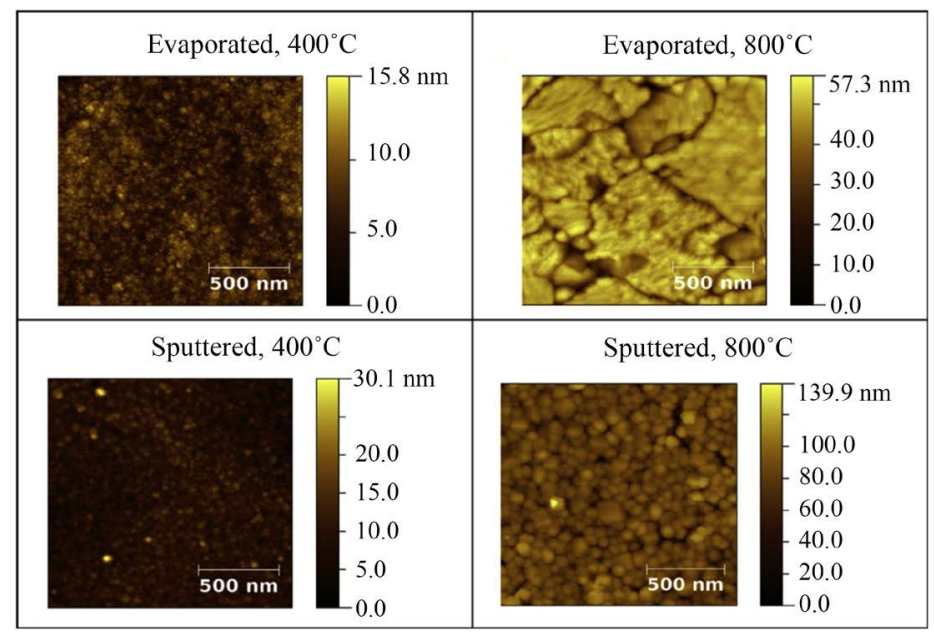

Figure 7. The evaporated thin films are much smoother than the sputtered thin films [17].

sub-micron silver regions distributed in a PMMA matrix. Films were found to be electrically conducting where a connected silver network was there. The sheet resistance of the PMMA-Ag nanocomposite was around $4500 \Omega$ /sheet which is 11 orders of magnitude lower than the sheet resistance of PMMA $\left(10^{15} \Omega\right)$. Thus, these films can be used for applications such as transparent large area collectors for solar cell, soft electrically conducting bio-electrical interfaces, etc. The spun coated films (Figure 7) and the solution cast films were found to be discrete silver islands which are electrically insulating. Therefore, by varying the solution evaporation rate, the properties and the morphology of the nanocomposite films can be controlled. Cytotoxicity studies show that the silver nanoparticles and the PMMA-Ag nanocomposite films are antibacterial in nature, where silver leads to complete elimination of $E$. coli colony forming units. Solution cast films containing the silver nanoparticles are also cytotoxic and inhibit bacterial growth. Also, the nanoparticle dispersed into pump oil and the thermal conductivity of the resultant mixture is measured. The thermal conductivity of the oil could be increased by over $50 \%$ by adding an extremely small fraction of the silver nanoparticles.

\subsection{Dispersion of Metals into Polymers}

Toxic nature of copper and silver even at low concentrations found applications as antimicrobial in agriculture, healthcare, and the industry in general. These metals are stable under conditions currently found in the industry used as additives. These metal based additives are found as particles, ions absorbed/exchanged in different carriers, salts, hybrid structures, etc. By incorporating these metals as nanoparticles into polymer matrices have many antimicrobial applications. These polymer/metal nanocomposites can be prepared by several routes such as in situ synthesis of the nanoparticle within a hydrogel or direct addition of the metal nanofiller into a thermoplastic matrix [18]. The former approach is mostly used for polymer hydrogel nanocomposites where the 
presence in the macromolecules of both several functional groups and a water-rich medium improves the metal stabilization and dispersion. The second approach is currently used in thermoplastic composites where the high viscoelastic matrix at the melt state improves the dispersion of the nanoparticles. A relevant set of antimicrobial polymer/copper nanocomposites are based on natural occurring biopolymers such as cellulose. Nanocomposites based on both vegetable and bacterial cellulose matrices were prepared by in situ and ex situ methods. The results show that the chemical nature and morphology of the copper nanofillers have great effect on the antibacterial activity. An increase in the antibacterial activity is found with the increasing copper content in the composites. The cellulosic matrices also show an effect on the antibacterial efficiency of the nanocomposites, by acting as the most effective substrate with vegetal cellulose fibers.

\subsection{Sol-Gel Method}

The one of the important method is sol-gel method. The term sol-gel is associated to two reaction steps, sol and gel. Sol is a colloidal suspension of solid particles in a liquid phase and gel the interconnected network formed between phases [8].

The sol-gel technique (Figure 8) allows engineers and scientists to design a new generation of advanced materials with unique properties. Though, it is a very old method to prepare functional materials since the discovery of the "water glass" by Von Helmont. However, sol-gel method got scientific relevance later by the preparation of silica gel. The sol-gel process consists in two main reactions: hydrolysis and condensation. Both are multi-steps processes and occur sequentially. Hydrolysis is a cleaving of organic chain bonding to metal and subsequent replacement with - $\mathrm{OH}$ groups through nucleophilic addition. The protonated species leaves the hydrolysed metal as an alcohol (alcoxolation). The metal reactivity, amount of water, solvent, temperature and the use of complex agents or catalysts are the main reaction parameters. To use or not to use a catalyst depends on the chemical nature of the metal atom and steric hindrance of the alkoxide group. Electrophilic character of the metal atom and its ability to increase the coordination number seems to be the main parameters. One of the most interesting advantages of the use of sol-gel method is its compatibility with polymers and polymerization processes, which allows the formation of nanoparticles in the presence of organic molecules.

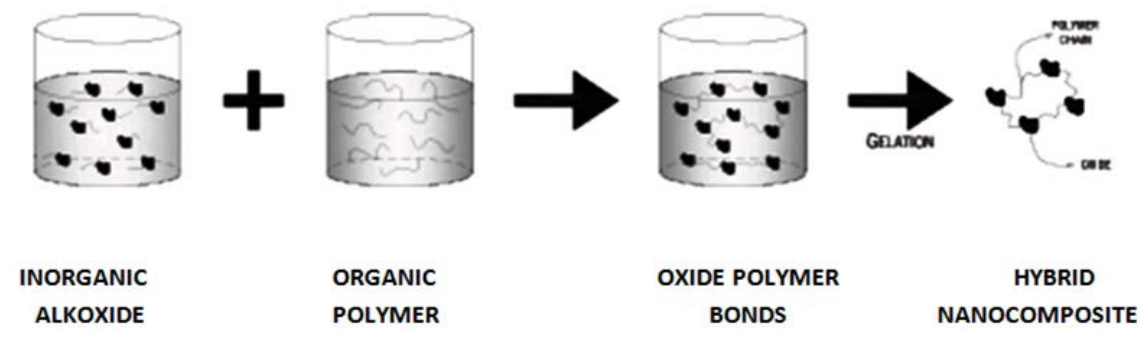

Figure 8. The process of polymerization in Sol-gel Method [8]. 


\subsubsection{Evaporation of Metal onto Polymer Substrate}

As discussed earlier, the possibility of metal polymer interaction is very weak. Also, the cohesive energy of polymers is two orders of magnitude lower than the cohesive energy of metals. As a result the metals do not wet untreated polymer surfaces and form cluster during thermal evaporation deposition. These metal particles coalesce and form a continuous film on polymer surfaces. The probability of absorption of metal atoms has been described through the condensations coefficient, $\mathrm{C}$ which is defined as the ratio of the number of adsorbed metal atoms to the total number of metal atoms arriving at the surface. The condensation coefficient is very important to estimate the influence of different surface treatments on the adhesion of metals on polymers. In this method, polymer films on substrate are prepared by sputtering or solution cast then metals are evaporated in vaccum of high order. The deposition rate and thickness is monitored by a quartz crystal. The nominal or equivalent thickness is a measure for the amount of metal evaporated onto the surface, which is not necessarily identical to the amount of metal actually deposited onto the surface. The latter can be much lower depending on the condensation coefficient which is usually unknown, and needs to be determined within the experiment itself. As mentioned above, the condensation coefficient, $\mathrm{C}$ is defined as the ratio of the number of atoms actually deposited onto the surface to the total number of atoms arriving at the surface. It was shown that the morphology of the films and C are correlated through the nucleation process. The decrease in the condensation coefficient with temperature was related to morphological changes occurring at the metal/polymer interface [19].

\subsubsection{Thermal Vacuum Evaporation}

Polymer matrices have been frequently used as particle stabilizers in chemical synthesis of metal colloids since these prevent agglomeration of the particles. Conventional ex situ methods involved the process where toxicating and potentially hazardous reactants are used. Also, it is difficult to disperse silver nanoparticles homogeneously into a polymer matrix by ex situ methods because of the easy agglomeration of nanoparticles. At present, it is possible to obtain nanoparticles of different shape and size in polymeric environment using various polymeric systems and different approaches. Increasing environmental concerns over synthesis route resulted in an attempt to adopt eco friendly methods. One of them is a simplest and eco friendly technique to form such particulate structures which are generally known as island or discontinuous metal films, through vacuum evaporation of metal on to a dielectric substrate by stopping the deposition at a very early stage.

Silver films on PVP and PS show room temperature resistance which are equal to substrate resistance due to formation of large silver clusters separated by large distances. But silver films on P4VP show negative TCR at higher temperatures and almost zero TCR at lower temperatures. Also, the resistance of silver films on P4VP was found to be in the order of few tens of mega ohms. The morphology of the particulate film is directly related to the electrical behavior of 
silver films on polymer substrate. For manipulating the size and distribution of silver clusters deposited on polymer matrices both blending and thickness of metal film can be used as tools to tailor and optimize the nanocomposite structure. We have carried out vacuum deposition of thin discontinuous silver films on the composites of Polystyrene (PS), Poly (vinylpyrrolidone) (PVP), Poly (2-vinylpyridine) (P2VP) and Poly (4-vinylpyridine) (P4VP) by one of the simplest techniques, vacuum evaporation of metal on to a dielectric substrate by stopping the deposition at a very early stage. These organized small metal particles with an average inter- particle separations of a couple of nm exhibit interesting optical and electrical properties. The oxidation of islands causes an irreversible increase in electrical resistance. The morphology of sub-surface particulate structures also depends upon polymer metal interaction along with filling factor. The morphology and formation of such structures depend on thermodynamic as well as deposition parameters. A completely submerged particle structure is thermodynamically favourable provided the inequality

$$
\gamma 1>\gamma 2+\gamma 12
$$

$\gamma 1 \& \gamma 2$-Surface tension of the particle \& substrate material. $\gamma 12$ is the interfacial tension.

Silver deposited on PS and PVP forms highly agglomerated subsurface particulate structure with large separations between the metal particles. Silver films on metal interacting polymers ( $\mathrm{P} 4 \mathrm{VP}$ and $\mathrm{P} 2 \mathrm{VP}$ ) have room temperature resistance in the range of a few tens to a few hundred $\mathrm{M} \Omega$ /sheet, which is desirable for device applications. Also, P4VP much interacting due to para position of $\mathrm{Ni}$ trogen in its pyridine ring. Silver films of various thicknesses were deposited on these substrates coated with PS/P2VP/P4VP \& PVP/P4VP polymer composites held at $457 \mathrm{~K}$ in a vacuum better than $8 \times 10^{-6}$ Torr. Blending has positive effect on electrical properties of silver films deposited on these blends. This is an approach to produce stable island films with reasonable control over their electrical resistance. Higher thickness films show almost zero TCR near room temperature, a desirable property for most of the devices. Low thickness films show a negative TCR, characteristic of island films. Optical absorption spectra of films show shift towards longer wavelengths. The morphology of silver particulate films modify with P4VP/P2VP content in the blends. The increase in the thickness of silver deposited on a particular blend results in a reduction of inter-particle separation and increase in mean particle size (Figures 9(a)-(c)) [5] [20].

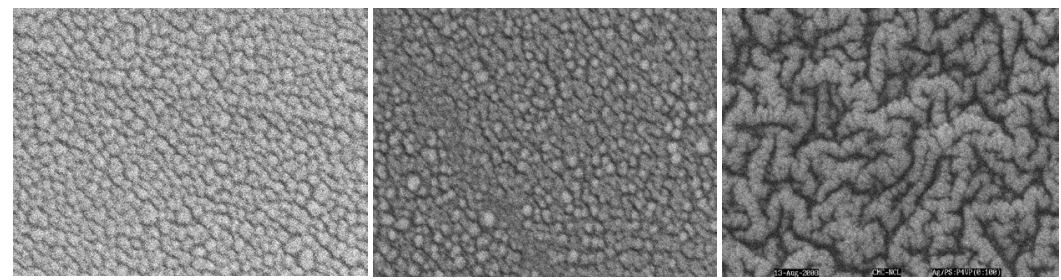

Figure 9. (a) PS:P4VP(100:0), particle size 92 nm; (b) PS:P4VP(50:50), particle size $\sim 80 \mathrm{~nm}$; (c) PS:P4VP(0:100), particle size $\sim 58 \mathrm{~nm}$. Thickness of film-50 nm. 


\section{Conclusions}

In the chemical reduction method, the size of nanoparticles is small around 12 $\mathrm{nm}$. Charecterisation process leads to confirmation of silver embedded into polymer composite. But the agglomeration of nanoparticles does not give stability in the result.

Co-Condensation (COCON) technique has the advantages of chemical and vacuum routes for synthesis of nanoparticle. In this method, pure, small nanoparticles with a narrower particle size distribution are produced without getting oxidised due to solvent. This solvation effect is very important as it disperses the nanoparticles within the liquid medium. This prevents oxidation of the nanoparticles which is a common problem in the synthesis of metal nanoparticles.

In spin coating and solutions, cast nanoparticles are dispersed over a wide area without agglomeration. In this metho, discrete films are formed which are electrically conducting.

Dispersion of nanoparticles can be prepared by several routes such as in situ synthesis of the nanoparticle within a hydrogel or direct addition of the metal nanofiller into a thermoplastic matrix. These have vast application in the field of antimicrobial. Whenever, the application in the form of thin films is not possible.

Besides the fact that sol-gel method is very old method of preparation of nanoparticle, it is still advantageous due to compatibility with polymers.

Thermal evaporation of metals on polymers is more effective because cohesive energy of polymers is less than the cohesive energy of metals. The interaction of metal with polymer is very bleak. The metals do not wet untreated polymers and coalesce by forming a continuous film on polymer surfaces. Polymer matrices prevent the agglomeration of nanoparticles. Further embedment of nanoparticle into polymers can be done by using special type of polymers. As silver films on P4VP show negative TCR at higher temperatures and almost zero TCR at lower temperatures. Also, the resistance of silver films on P4VP was found to be in the order of few tens of mega ohms. P4VP is much interacting due to para position of Nitrogen in its pyridine ring. Thus, the morphology of sub-surface particulate structures depends upon polymer metal interaction along with filling factor. Further, the size and inter space distribution of nanoparticles can be modified by polymer composites. The silver embedded into Polyvinylpyrrodine film prepared by this method can find many antimicrobial applications.

A variety of routes to prepare nano-polymer composites with great homogeneity depend on the nature of inorganic particles and properties. The main criteria are to find out best between the polymer matrix and the dispersed particles. There is no route to prepare homogeneous material, but needs to optimize the required properties.

\section{Acknowledgements}

I acknowledge with a huge thank to all the authors whose work I refer for my re- 
view article.

\section{Conflicts of Interest}

The author declares no conflicts of interest regarding the publication of this paper.

\section{References}

[1] Mittal, V. (2010) Optimization of Polymer Nanocomposite Properties. Wiley-VCH, Weinheim, 1-19.

[2] Majhi, S.M., Naik, G.K., Lee, H.J., Song, H.-G., Lee, C.-R., Lee, I.-H. and Yu, I.-T. (2018) Au@NiO Core-Shell Nanoparticles as a p-Type Gas Sensor: Novel Synthesis, Characterization, and Their Gas Sensing Properties with Sensing Mechanism. Sensors \& Actuators B: Chemicals, 268, 223-231.

https://doi.org/10.1016/j.snb.2018.04.119

[3] Hassan, K. and Siavash, I. (2012) Silver Nanoparticles. IntechOpen Publication, Rijeka, 1-35.

[4] Ajayan, P.M. (2004) Bulk Metal and Ceramics Nanocomposites. In: Ajayan, P.M., Schadler, L.S. and Braun, P.V., Eds., Nanocomposite Science and Technology, Wiley-VCH, Hoboken, 1-75. https://doi.org/10.1002/3527602127.ch1

[5] Parashar, P. (2015) Preparation and Characterization of Subsurface Silver Particulate Films on Polymer Blends of Polystyrene/Poly(2-vinylpyridine)/Poly(vinylpyrollidone)/Poly(4-vinylpyridine). Soft Nanoscience Letters, 5, 3-11. https://doi.org/10.4236/snl.2015.51002

[6] Parashar, P. (2013) Synthesis of Silver Nanocomposite with Poly(vinylpyrollidone) and Poly(4-vinylpyridine) for Antimicrobial Activity. Advanced Materials Research, 772, 9-14. https://doi.org/10.4028/www.scientific.net/AMR.772.9

[7] Parashar, P. (2014) Synthesis of Silver Nanocoposite with Eudragit Rl 100 for the Drug Delivery System. International Journal of Education \& Applied Sciences Research, 1, 51-54.

[8] Oliveira, M. and Machado, A.V. (2013) Preparation of Polymer-Based Nanocomposites by Different Routes. 1-22.

https://repositorium.sdum.uminho.pt/bitstream/1822/26120/1/Chapter.pdf

[9] Wankhede, Y.B., Kondawar, S.B., Thakare, S.R. and More, P.S. (2013) Synthesis \& Characterisation of Silver Nanoparticles Embedded in Polyaniline Nanocomposite. Advanced Materials Letters, 4, 89-93. https://doi.org/10.5185/amlett.2013.icnano.108

[10] Igoris, P., Judita, P., Asta, G., Sigitas, T. and Ramūnas, N. (2007) Investigation of Silver Polymer Nanocomposites. Materials Science (Medžiagotyra), 13, 188-192.

[11] Biswas, A., Aktas, O.C., Kanzow, J., Saeeda, U., Strunskus, T., Zaporojtchenko, V. and Faupel, F. (2004) Polymer-Metal Optical Nanocomposites with Tunable Particle Plasmon Resonance Prepared by Vapor Phase Co-Deposition. Materials Letters, 58, 1530-1534. https://doi.org/10.1016/j.matlet.2003.10.037

[12] Charton, F.M. (2003) Growth of Ag on PET Deposited by Magnetron Sputtering. Vacuum, 68, 65-73. https://doi.org/10.1016/S0042-207X(02)00289-0

[13] Bold, H., Umeda, N., Plaskin, O.A., Takeda, Y. and Kishimoto, N. (2005) High-Fluence Implantation of Negative Metal Ions into Polymers Surface Modification and Nanoparticle Formation. Surface and Coatings Technology, 196, 373-377. 
https://doi.org/10.1016/j.surfcoat.2004.08.159

[14] Sun, Y., Gates, B., Mayers, B. and Xia, Y. (2002) Crystalline Silver Nanowires by Soft Solution Processing. Nano Letters, 2, 165-168. https://doi.org/10.1021/nl010093y

[15] Mailard, M., Huang, O. and Brus, L. (2003) Silver Nanodisk Growth by Surface Plasmon Enhanced Protoreduction of Adsorbed [Ag+]. Nano Letters, 3, 1611-1615. https://doi.org/10.1021/nl034666d

[16] Picu, R.V. and Ozmusul, M.S. (2002) Enhanced Structure at the Interface between the Polymer Matrix and Spherical Nanopartilces in Polymer Based Nanocomposites. Technical Proceedings of the 2002 International Conference on Computational Nanoscience and Nanotechnology, Vol. 2, 399-402.

[17] Anshu, A., Pradhan, S., Shah, I. and Lisa, P. (2003) Synthesis and Characterization of Metal Nanoparticles and the Formation of Metal-Polymer Nanocomposites. Materials Research Society Symposium Proceedings, 740, 179-184. https://doi.org/10.1557/PROC-740-I6.10

[18] Humberto, P. (2015) Antimicrobial Polymers with Metal Nanoparticles. International Journal of Molecular Sciences, 16, 2099-2116.

https://doi.org/10.3390/ijms16012099

[19] Zaporojtchenko, Y., Erichsen, J., Zekonyte, A., T'hran, T., Stn-rnskusad and Faupel, F. (2002) Adsorption of Atoms on Polymers Metallization of Polymers. Kluwer Academic/Plenum Publishers, Dordrecht.

[20] Parashar, P. (2013) Silver Particulate Films on Compatible Softened Polymer Composites. In: Applications of Calorimetry in a Wide Context-Differential Scanning Calorimetry, Isothermal Titration Calorimetry and Microcalorimetry, Intech Publication, London, Chapter 10, 221-251. https://doi.org/10.5772/54502 